\title{
Pulsation properties of ultra-massive DA white dwarf stars with ONe cores
}

\author{
Francisco C. De Gerónimo ${ }^{1,2}$, Alejandro H. Córsico ${ }^{1,2}$, Leandro G. Althaus ${ }^{1,2}$, \\ Felipe C. Wachlin ${ }^{1,2}$, and María E. Camisassa ${ }^{1,2}$ \\ ${ }^{1}$ Grupo de Evolución Estelar y Pulsaciones, Facultad de Ciencias Astronómicas y Geofísicas, Universidad Nacional de La Plata, \\ Paseo del Bosque s/n, 1900 La Plata, Argentina \\ e-mail: fdegeronimo@fcaglp.unlp.edu.ar \\ 2 Instituto de Astrofísica La Plata, IALP (CCT La Plata), CONICET-UNLP, Argentina
}

Received 6 July 2018 / Accepted 27 August 2018

\begin{abstract}
Context. Ultra-massive hydrogen-rich white dwarf stars are expected to harbor oxygen/neon cores resulting from the progenitor evolution through the super-asymptotic giant branch phase. As evolution proceeds during the white dwarf cooling phase, a crystallization process resulting from Coulomb interactions in very dense plasmas is expected to occur, leading to the formation of a highly crystallized core. In particular, pulsating ultra-massive white dwarfs offer a unique opportunity to infer and test the occurrence of crystallization in white dwarf interiors as well as physical processes related with dense plasmas.

Aims. We aim to assess the adiabatic pulsation properties of ultra-massive hydrogen-rich white dwarfs with oxygen/neon cores.

Methods. We studied the pulsation properties of ultra-massive hydrogen-rich white dwarf stars with oxygen/neon cores. We employed a new set of ultra-massive white dwarf evolutionary sequences of models with stellar masses in the range $1.10 \leq M_{\star} / M_{\odot} \leq 1.29$ computed by taking into account the complete evolution of the progenitor stars and the white dwarf stage. During the white dwarf cooling phase, we considered element diffusion. When crystallization set on in our models, we took into account latent heat release and also the expected changes in the core chemical composition that are due to phase separation according to a phase diagram suitable for oxygen and neon plasmas. We computed nonradial pulsation $g$-modes of our sequences of models at the ZZ Ceti phase by taking into account a solid core. We explored the impact of crystallization on their pulsation properties, in particular, the structure of the period spectrum and the distribution of the period spacings.

Results. We find that it would be possible, in principle, to discern whether a white dwarf has a nucleus made of carbon and oxygen or a nucleus of oxygen and neon by studying the spacing between periods.

Conclusions. The features found in the period-spacing diagrams could be used as a seismological tool to discern the core composition of ultra-massive ZZ Ceti stars, this should be complemented with detailed asteroseismic analysis using the individual observed periods.
\end{abstract}

Key words. stars: evolution - stars: oscillations - stars: interiors - white dwarfs

\section{Introduction}

White dwarf (WD) stars are the final stage of evolution for low- and intermediate-mass stars. Most of these stars are postasymptotic giant branch (AGB) remnants with carbon-oxygen cores and stellar masses $M_{\mathrm{wd}} \sim 0.6 M_{\odot}$. The mass distribution also exhibits a tail of massive WDs peaking at $\sim 0.82 M_{\odot}$, (Kleinman et al. 2013). The existence of massive WDs with $M_{\text {wd }} \gtrsim 0.8 M_{\odot}$ (Castanheira et al. 2010, 2013) and ultra-massive WDs with $M_{\mathrm{wd}} \gtrsim 1.15 M_{\odot}$, (Hermes et al. 2013; Curd et al. 2017) is also revealed.

Theoretical studies suggest that progenitor stars that are initially more massive than $\sim 7 M_{\odot}$ are expected to evolve through the super-AGB (S-AGB) phase, igniting carbon in their interiors and eventually resulting in the formation of oxygen and neon $(\mathrm{ONe})$ WDs with masses $M_{\mathrm{wd}} \gtrsim 1.05 M_{\odot}$ (García-Berro et al. 1997). Specifically, carbon is ignited in semi-degenerate conditions, leading to repeated carbon-burning shell flashes that propagate inward, turning the $\mathrm{CO}$ core into an $\mathrm{ONe}$ mixture (Garcia-Berro \& Iben 1994; Siess 2006). After carbon is exhausted in the core, and as a result of mass loss, the remnant evolves toward the WD domain, and a WD is formed with a core made mostly of ${ }^{16} \mathrm{O}$ and ${ }^{20} \mathrm{Ne}$ with traces of ${ }^{23} \mathrm{Na}$ and ${ }^{24} \mathrm{Mg}$ (Siess 2007).

As is well known, many WDs exhibit multiperiodic luminosity variations caused by pulsations. In particular, variable H-rich (DA) WDs, called ZZ Ceti (or DAV), are the most numerous class of compact pulsators. These stars show variations in their luminosity in a narrow instability strip of $10500 \lesssim$ $T_{\text {eff }} \lesssim 12300 \mathrm{~K}$ (Fontaine \& Brassard 2008; Winget \& Kepler 2008; Althaus et al. 2010a) with photometric variations of up to $0.3 \mathrm{mag}$. These variations are the result of spheroidal non-radial $g$-mode pulsations with low harmonic degree $(\ell \leq 2)$ and periods in the range 70-1500 s. In particular, the first attempt of studying the adiabatic pulsational properties of ultra-massive ONe-core WDs was made by Córsico et al. (2004), who demonstrated that pulsational expectations such as the forward and mean period spacing of ONe WDs are markedly different from those of CO WDs.

One interesting point in studying ultra-massive pulsating DA WDs lies in the fact that these stars are expected to harbor a crystallized core resulting from Coulomb interactions in very dense plasmas. Although the occurrence of crystallization in WDs was theoretically suggested several decades ago by Kirzhnits (1960), 
Abrikosov (1961), Salpeter (1961) and van Horn (1968; see also recent studies by Montgomery \& Winget 1999; Metcalfe et al. 2004; Córsico et al. 2005; Brassard \& Fontaine 2005), it was not until quite recently that the existence of crystallized WDs was inferred from the study of WD luminosity function of stellar clusters (Winget et al. 2009; García-Berro et al. 2010). The fact that ultra-massive pulsating DA WDs are expected to be crystallized turns these stars into unique objects from which the occurrence of crystallization in WD interiors can also be inferred. The first star studied in this sense was the ultra-massive ZZ Ceti star BPM 37093 (Kanaan et al. 1992, 2005), which is expected to have a partially crystallized core (Metcalfe et al. 2004; Brassard \& Fontaine 2005).

We here aim to assess the adiabatic pulsation properties of ultra-massive $\mathrm{H}$-rich WDs with $\mathrm{ONe}$ cores on the basis of full evolutionary models that incorporate the most updated physical ingredients governing the progenitor and WD evolution. This investigation constitutes a substantial improvement over the study of Córsico et al. (2004) in three major aspects: first, the chemical profiles for all of our WD models, which were taken from Siess (2010), are consistent with the predictions of the progenitor evolution with stellar masses in the range $9.0<$ $M_{\text {ZAMS }} / M_{\odot}<10.5$ from the zero-age main sequence (ZAMS) to the end of the S-AGB phase. Thus, not only a realistic ONe inner distribution expected for each WD mass is considered in the WD modeling, but also realistic chemical profiles and inter-shell masses built up during the S-AGB are taken into account. Second, we take into account for the first time the changes in the core chemical composition resulting from phase separation as WDs crystallize. To this end, we consider phase diagrams suitable for ${ }^{16} \mathrm{O}$ and ${ }^{20} \mathrm{Ne}$ plasmas (Medin \& Cumming 2010). We show that the changes in the chemical profiles resulting from phaseseparation processes leave strong signatures in the theoretical pulsational spectrum and must be taken into account in realistic computations of the pulsational properties of ultra-massive WDs. Finally, element diffusion was included for all model sequences, from the beginning of the WD cooling track. Element diffusion smoothes the inner chemical profiles, thus altering the run of the Brunt-Väisälä frequency, and hence the period spectrum and mode-trapping properties. The pulsational study presented in this work is based on evolving models of ultra-massive WDs recently computed in Camisassa et al. (2018). These evolutionary sequences that incorporate all these improvements were computed from the very beginning of the cooling track down to very low surface luminosities. The paper is organized as follows. A summary of the numerical codes and the treatment of crystallization we employed is provided in Sect. 2. In Sect. 3 we briefly describe the evolutionary models and the core chemical redistribution that is due to phase separation during crystallization. In Sect. 4 we present a detailed description of the pulsation computations. In particular, in Sect. 4.1 we assess the impact of crystallization on the pulsation spectrum of our ONe-core WD models, and in Sect. 4.2 we compare the pulsation properties of our ONe-core WD models with those of CO-core WDs for a fixed mass value. Finally, in Sect. 5 we summarize the main findings of this work.

\section{Numerical tools}

\subsection{Evolutionary code}

The DA WD evolutionary models developed in this work were computed with the LPCODE evolutionary code (see Althaus et al. 2005, 2010b; Renedo et al. 2010; Romero et al.
2012; Miller Bertolami 2016, for detailed physical description). This numerical tool has been employed to study various aspects of the evolution of low-mass stars (Wachlin et al. 2011; Althaus et al. 2013, 2015), the formation of horizontal branch stars (Miller Bertolami et al. 2008), extremely lowmass WDs (Althaus et al. 2013), AGB, and post-AGB evolution (Miller Bertolami 2016), among others. More recently, the code has been used to assess the impact of the uncertainties in progenitor evolution on the pulsation inferences of ZZ Ceti stars (De Gerónimo et al. 2017, 2018). Next we describe the main input physics of the code that are relevant for computing the ultra-massive WD models: (i) convection is treated according to the mixing length formulation (ML2, Tassoul et al. 1990); (ii) radiative and conductive opacities are those from OPAL (Iglesias \& Rogers 1996) and Cassisi et al. (2007), respectively; (iii) the molecular radiative opacities for the low-temperature regime are those from Ferguson et al. (2005); (iv) the equation of state for the low-density regime is taken from Magni \& Mazzitelli (1979) and that for the highdensity regime from Segretain et al. (1994), which accounts for both the solid and liquid phases; (v) element diffusion, including gravitational settling and chemical and thermal diffusion, is considered; and (vi) energy release from crystallization (latent heat and gravitational energy associated with $\mathrm{ONe}$ phase separation) are included by considering a phase diagram that is suitable for the dense ONe interiors of ultra-massive WD (Medin \& Cumming 2010). To our knowledge, this is the first pulsational analysis of ultra-massive WD models that includes the phase-separation processes in $\mathrm{ONe}$ cores.

\subsection{Treatment of crystallization and phase separation}

Cool WD stars are expected to crystallize as a result of strong Coulomb interactions in their very dense interior (van Horn 1968). Crystallization sets in when the energy of the Coulomb interaction between neighboring ions is much higher than their thermal energy. This occurs when the ion coupling constant $\Gamma \equiv\left\langle Z^{5 / 3}\right\rangle \mathrm{e}^{2} / a_{\mathrm{e}} k_{\mathrm{B}} T$ is larger than a certain value, which depends on the adopted phase diagram. Here $a_{\mathrm{e}}$ is the interelectronic distance, $\left\langle Z^{5 / 3}\right\rangle$ is an average (by number) over the ion charges, and $k_{\mathrm{B}}$ is Boltzmann's constant. The other symbols have their usual meaning. The occurrence of crystallization leads to two additional energy sources: the release of latent heat, and the release of gravitational energy associated with changes in the chemical composition profile induced by crystallization (Garcia-Berro et al. 1988a,b; Winget et al. 2009). In our study, these two additional energy sources are included selfconsistently and locally coupled to the full set of equations of stellar evolution. In particular, the luminosity equation is appropriately modified to account for both the local contribution of energy released from the core chemical redistribution and the latent heat. At each time step, the crystallization temperature and the change in chemical profile resulting from phase separation are computed using the appropriate phase diagram. In particular, the oxygen-enhanced convectively unstable liquid layers overlying the crystallizing core are assumed to be instantaneously mixed, a reasonable assumption considering the long evolutionary timescales of WDs. The chemical redistribution due to phase separation and the associated release of energy have been considered following the procedure described in Althaus et al. (2010c), appropriately modified by Camisassa et al. (2018) for ONe plasmas. To assess the enhancement of ${ }^{20} \mathrm{Ne}$ in the crystallized core, we used the azeotropic-type phase diagram of Medin \& Cumming (2010). It is worth mentioning that this is 
a two-component phase diagram, even though our WD models contain trace amounts of other elements in the crystallized regions. After computing the chemical composition of both the solid and the liquid phases, we evaluated the net energy released in the process as in Althaus et al. (2010c); see also Isern et al. (1997).

\subsection{Pulsation code}

We computed nonradial $g$-mode pulsations of our complete set of ultra-massive ONe-core DA WD models using the adiabatic version of the LP-PUL pulsation code described in Córsico \& Althaus (2006). The pulsation code is based on the general Newton-Raphson technique that solves the full fourthorder set of equations and boundary conditions governing linear, spheroidal, adiabatic, nonradial stellar pulsations following the dimensionless formulation of Dziembowski (1971). We did not consider torsional modes, since these modes are characterized by very short periods (up to $20 \mathrm{~s}$; see Montgomery \& Winget 1999) and are not observed in ZZ Ceti stars. To account for the effects of crystallization on the pulsation spectrum of $g$-modes, we adopted the "hard sphere" boundary conditions, which assume that the amplitude of the eigenfunctions of $g$-modes is drastically reduced below the solid/liquid interface because of the nonshear modulus of the solid, as compared with the amplitude in the fluid region (see Montgomery \& Winget 1999). In our code, the inner boundary condition is not the stellar center, but instead the mesh-point corresponding to the crystallization front moving toward the surface (see Córsico et al. 2004, 2005; Romero et al. 2013). Specifically, the hard-sphere boundary condition at the radial shell corresponding to the outward-moving crystallization front $\left(r_{\mathrm{c}}=r\left(M_{\mathrm{c}}\right)\right)$ reads

$y_{1}=0, \quad y_{2}=$ arbitrary, $\ell y_{3}-y_{4}=0$.

Here $y_{1}$ and $y_{2}$ represent the radial and horizontal displacements, respectively, and $y_{3}$ and $y_{4}$ are the Eulerian perturbation of the gravitational potential and its derivative. The last condition is the same as for the normal case in which the core is in a fluid state and the boundary condition is applied at the stellar center; see Appendix B of Montgomery \& Winget (1999).

The asymptotic period spacing is computed as in Tassoul et al. (1990). For $g$-modes with high radial order $k$ (long periods), the separation of consecutive periods $(|\Delta k|=1)$ becomes nearly constant at a value given by the asymptotic theory of nonradial stellar pulsations. Specifically, the asymptotic period spacing is given by

$\Delta \Pi_{\ell}^{a}=\Pi_{0} / \sqrt{\ell(\ell+1)}$,

where

$\Pi_{0}=2 \pi^{2}\left[\int_{r_{1}}^{r_{2}} \frac{N}{r} \mathrm{~d} r\right]^{-1}$.

The squared Brunt-Väisälä frequency $(N$, one of the critical frequencies of nonradial stellar pulsations) is computed as

$N^{2}=\frac{g^{2} \rho}{P} \frac{\chi_{T}}{\chi_{\rho}}\left[\nabla_{\mathrm{ad}}-\nabla+B\right]$,

where the compressibilities are defined as (Tassoul et al. 1990; Brassard et al. 1991)

$\chi_{\rho}=\left(\frac{\partial \ln P}{\partial \ln \rho}\right)_{T,\left\{X_{i}\right\}} \chi_{T}=\left(\frac{\partial \ln P}{\partial \ln T}\right)_{\rho,\left\{X_{i}\right\}}$.
The Ledoux term $B$ is computed as

$B=-\frac{1}{\chi_{T}} \sum_{1}^{M-1} \chi_{X_{i}} \frac{\partial \ln X_{i}}{\partial \ln P}$,

where

$\chi_{X_{i}}=\left(\frac{\partial \ln P}{\partial \ln X_{i}}\right)_{\rho, T,\left\{X_{j \neq i}\right\}}$.

The computation of the Ledoux term $B$ has been generalized in order to include the effects of having multiple chemical species $\left({ }^{1} \mathrm{H},{ }^{4} \mathrm{He},{ }^{12} \mathrm{C},{ }^{16} \mathrm{O},{ }^{20} \mathrm{Ne},{ }^{23} \mathrm{Na},{ }^{24} \mathrm{Mg}\right)$ that vary in abundance.

When a fraction of the WD core is crystallized, the lower limit of the integral in Eq. (3) coincides with the radius of the crystallization front $\left(r_{1}=r_{\mathrm{c}}\right)$, which moves outward as the star cools down and the fraction of crystallized mass increases. Hence, the integral in Eq. (3) decreases, leading to an increase in the asymptotic period spacing (Eq. (2)) and also in the periods themselves.

\section{Evolutionary models}

We computed the evolution and pulsation properties of four ultra-massive WD sequences with stellar masses $M_{\star}=$ $1.10,1.16,1.22$, and $1.29 M_{\odot}$ resulting from the complete evolution of the progenitor stars through the S-AGB phase. The core and inter-shell chemical profiles of our models at the start of the WD cooling phase were obtained from Siess (2010). The cores are composed mostly of ${ }^{16} \mathrm{O}$ and ${ }^{20} \mathrm{Ne}$ and smaller amounts of ${ }^{12} \mathrm{C},{ }^{23} \mathrm{Na}$, and ${ }^{24} \mathrm{Mg}$. Since element diffusion and gravitational settling operate throughout the WD evolution, our models develop pure hydrogen envelopes. In Table 1 we show the ${ }^{1} \mathrm{H}$ and ${ }^{4} \mathrm{He}$ mass content for each sequence, as well as the $T_{\text {eff }}$ and $\log g$ values corresponding to the onset of crystallization, and the fraction of crystallized mass at the boundaries of the ZZ Ceti instability strip. The He content of our WD sequences is given by the evolutionary history of progenitor star, but instead, the $\mathrm{H}$ content $\left(M_{\mathrm{H}} \sim 10^{-6} M_{\star}\right)$ has been set by imposing that the further evolution does not lead to $\mathrm{H}$ thermonuclear flashes on the WD cooling track. The higher mass model sequences start to crystallize at effective temperatures well above the instability strip, thus harboring a core that is almost completely crystallized at the time the sequences reach the $\mathrm{ZZ}$ Ceti stage. As consequence of element diffusion, the chemical profiles at low $T_{\text {eff }}$ are far smoother than those at the beginning of the WD stage (see Figs. 8 and 9 of Camisassa et al. 2018). In particular, the He buffer, which is located above the ONe core, is strongly eroded, therefore developing a region rich in ${ }^{4} \mathrm{He},{ }^{12} \mathrm{C},{ }^{16} \mathrm{O}$, and ${ }^{20} \mathrm{Ne}$.

Theoretical evidence suggests that if the core of a WD is initially composed of a mixture of ${ }^{16} \mathrm{O}$ and ${ }^{20} \mathrm{Ne}$ (Medin \& Cumming 2010), the crystallized region will have a higher abundance of ${ }^{20} \mathrm{Ne}$ than that in the original fluid state. On the other hand, the liquid regions overlying the crystallized ${ }^{20} \mathrm{Ne}$-enhanced layers will have a higher content of ${ }^{16} \mathrm{O}$. This region will become Rayleigh-Taylor unstable, since ${ }^{16} \mathrm{O}$ is less dense than ${ }^{20} \mathrm{Ne}$. This instability leads to a rehomogenization of the chemical profile in the fluid layers; as a result of this, the chemical abundances after crystallization differ substantially when compared with the initial ones. To compute the chemical rehomogenization, we employed the same algorithm as in Montgomery et al. (1999), Salaris et al. (1997), and Córsico et al. (2005). To derive the ${ }^{20} \mathrm{Ne}$ enhancement when 
Table 1. H and He mass content of our ONe-core ultra-massive DA WD models, together with the effective temperature and surface gravity at the onset of crystallization, and the fraction of crystallized mass at the blue and red edges of the ZZ Ceti instability strip.

\begin{tabular}{ccccccc}
\hline \hline$M_{\star} / M_{\odot}$ & $\begin{array}{c}M_{\mathrm{H}} / M_{\star} \\
\left(\times 10^{-6}\right)\end{array}$ & $\begin{array}{c}M_{\mathrm{He}} / M_{\star} \\
\left(\times 10^{-5}\right)\end{array}$ & $\begin{array}{c}T_{\text {eff }}^{\mathrm{c}} \\
(\mathrm{K})\end{array}$ & $\begin{array}{c}\log g^{\mathrm{c}} \\
(\mathrm{cgs})\end{array}$ & $\begin{array}{c}M_{\mathrm{c}} / M_{\star} \\
\left(T_{\text {eff }}=12500 \mathrm{~K}\right)\end{array}$ & $\begin{array}{c}M_{\mathrm{c}} / M_{\star} \\
\left(T_{\text {eff }}=10500 \mathrm{~K}\right)\end{array}$ \\
\hline 1.098 & 1.5 & 29.6 & 19881 & 8.83 & 0.81 & 0.92 \\
1.159 & 1.5 & 15.7 & 23291 & 8.95 & 0.90 & 0.96 \\
1.226 & 1.5 & 6.38 & 28425 & 9.12 & 0.96 & 0.98 \\
1.292 & 1.5 & 1.66 & 37309 & 9.33 & 0.994 & 0.998 \\
\hline
\end{tabular}

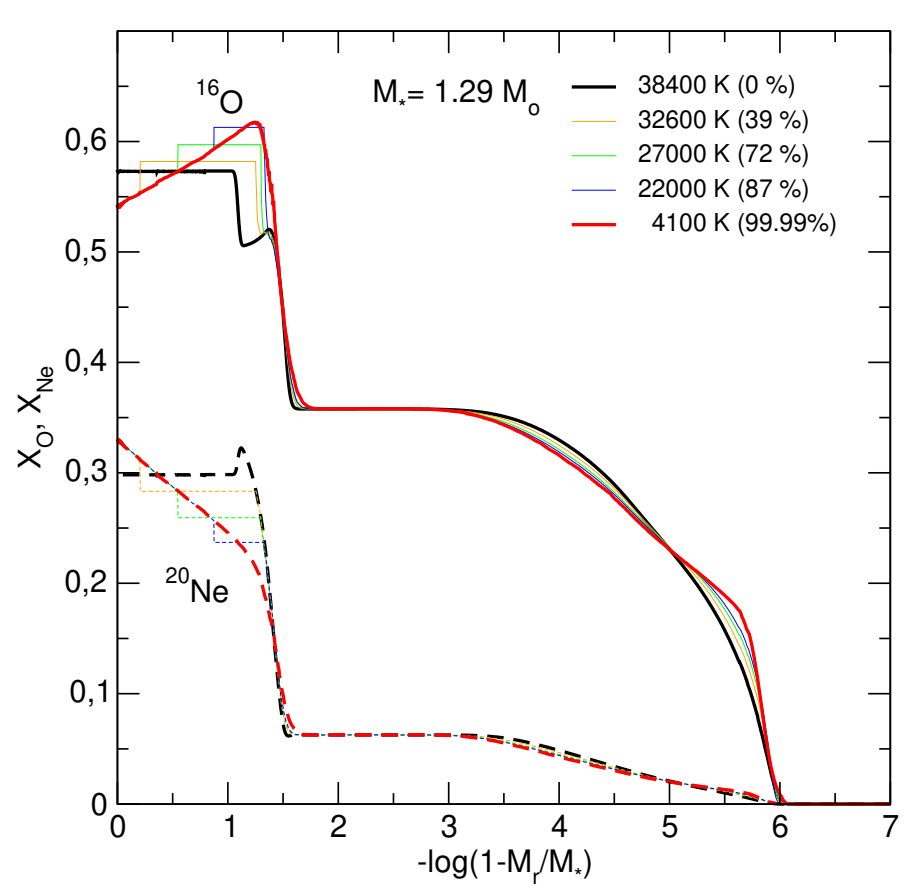

Fig. 1. Internal chemical profiles of ${ }^{16} \mathrm{O}$ (solid lines) and ${ }^{20} \mathrm{Ne}$ (dashed lines) in terms of the fractional mass for the $1.29 M_{\odot}$ ONe-core WD sequence corresponding to various percentages of crystallization. The thick black lines correspond to the profiles before crystallization, and the thin lines of different colors correspond to the chemical profiles resulting from chemical rehomogenization for different effective temperatures at increasing percentages of crystallized mass fraction, as indicated for some selected cases. The thick red curves correspond to a model with $T_{\text {eff }}=4046 \mathrm{~K}$ and a percentage of $99.99 \%$ of crystallized mass.

a given layer crystallizes, we adopted the Medin \& Cumming (2010) azeotropic-type phase diagram for a ${ }^{16} \mathrm{O} /{ }^{20} \mathrm{Ne}$ mixture. In our scheme for mixing, we first considered a crystallized, ${ }^{20} \mathrm{Ne}$-enhanced layer, and then we determined whether the innermost fluid shell had a higher oxygen content than the overlying one. If it did, we mixed the two fluid layers and performed the same comparison with the next layer farther out. In this way, we scanned outward through the fluid, and the process stopped when further mixing no longer decreased the ${ }^{20} \mathrm{Ne}$ content of the fluid between this point and the crystallization boundary. When the crystallization front moved outward due to cooling, the procedure was repeated.

In Fig. 1 we show the ${ }^{16} \mathrm{O}$ and ${ }^{20} \mathrm{Ne}$ chemical profiles in terms of the fractional mass for the $1.29 M_{\odot}$ ONe-core WD sequence corresponding to various degrees of crystallization. We did not include the trace elements $-{ }^{12} \mathrm{C},{ }^{23} \mathrm{Na}$, and ${ }^{24} \mathrm{Mg}$. Because we employed a two-component phase diagram, these minoritary elements are considered inert during crystallization. The shape of the chemical profile is modified, even in regions beyond the crystallization front. In particular, a pronounced step is formed in the chemical profiles at the border of the rehomogenized region.

\section{Pulsation calculations}

We computed adiabatic pulsation periods of $\ell=1,2 g$-modes in a range of periods covering the period spectrum that is typically observed in ZZ Ceti stars (70 s $\lesssim \Pi \lesssim 1500$ s). In our pulsation computations, we considered three situations:

1. Crystallization does not take place (NC case).

2. Crystallization occurs, but only latent heat is released, and the core chemical profile remains unaltered (LH case).

3. Crystallization does take place, involving a release of latent heat and also a chemical redistribution as a result of phase separation, as shown in Fig. 1 (LH+PS case).

The NC case is considered merely to assess the period spectrum of ultra-massive WDs when we neglect crystallization, that is, when the ordinary boundary conditions at the stellar center are adopted to solve the pulsation equations. The LH case is considered to show the pulsation properties of ultra-massive WDs when a solid core is included in computing the pulsational eigenspectra, that is, when we use the hard-sphere boundary conditions to compute the eigenfunctions of modes, but the core chemical profiles remain fixed as the WD crystallizes. Finally, we consider the LH+PS case, which constitutes the most physically plausible situation in which crystallization is considered and the core chemical profiles are being modified continuously during crystallization as a result of phase separation.

We show in Figs. 2-5 the chemical abundances by mass of ${ }^{1} \mathrm{H},{ }^{4} \mathrm{He},{ }^{12} \mathrm{C},{ }^{16} \mathrm{O},{ }^{20} \mathrm{Ne},{ }^{23} \mathrm{Na}$, and ${ }^{24} \mathrm{Mg}$ (upper panel), the Ledoux term $B$ (middle panel), and the logarithm of the squared Brunt-Väisälä $(N)$ and Lamb $\left(L_{\ell=1}\right)$ frequencies (lower panel) in terms of the outer mass fraction $\left(-\log \left(1-M_{\mathrm{r}} / M_{\star}\right)\right)$ for $\mathrm{ONe}$ core WD models with $M_{\star} / M_{\odot}=1.10,1.16,1.22$, and 1.29, respectively, and at an effective temperature in the middle of the ZZ Ceti instability strip $\left(T_{\text {eff }} \sim 11600 \mathrm{~K}\right)$. The models were computed taking into account crystallization with chemical redistribution that is due to phase separation ( $\mathrm{LH}+\mathrm{PS}$ case). The gray area marks the domain of crystallization, and the fraction of the crystallized mass is indicated for each model.

For all the stellar masses considered, a notorious feature of the core chemical profiles is the redistribution of ${ }^{16} \mathrm{O}$ and ${ }^{20} \mathrm{Ne}$ at the solid core that is due to phase separation, and the consequent chemical rehomogeneization at layers beyond the crystallization front, as can be seen in Fig. 1 for the case of the sequence with $M_{\star}=1.29 M_{\odot}$. The range of effective temperatures at which phase separation takes place depends on the stellar mass value. Specifically, phase separation during crystallization 

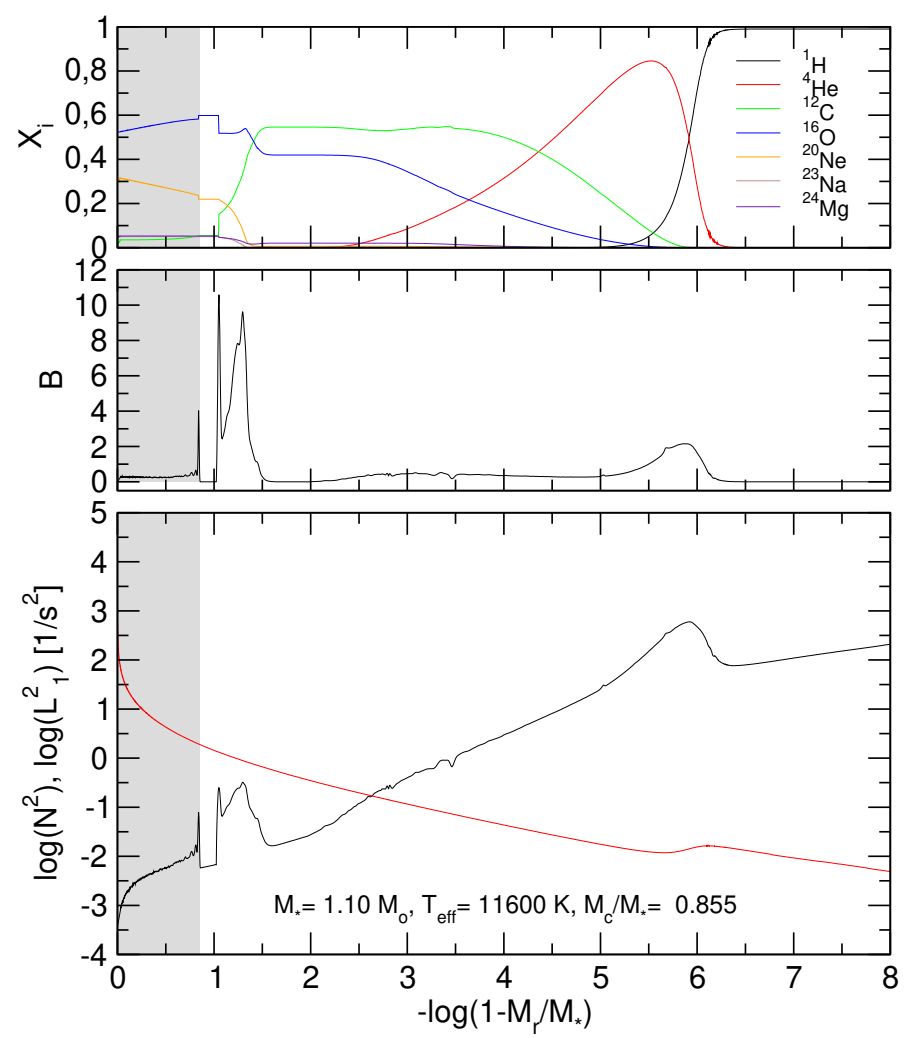

Fig. 2. Abundances by mass of ${ }^{1} \mathrm{H},{ }^{4} \mathrm{He},{ }^{12} \mathrm{C},{ }^{16} \mathrm{O},{ }^{20} \mathrm{Ne},{ }^{23} \mathrm{Na}$, and ${ }^{24} \mathrm{Mg}$ as a function of the fractional mass (upper panel), the Ledoux term $B$ (middle panel), and the logarithm of the squared Brunt-Väisälä and Lamb frequencies (lower panel), corresponding to an ONe-core WD model with $M_{\star}=1.10 M_{\odot}$ and $T_{\text {eff }} \sim 11600 \mathrm{~K}$. The model was computed taking into account latent heat release and chemical redistribution caused by phase separation during crystallization (LH+PS case). The gray area marks the domain of crystallization. $M_{\mathrm{c}} / M_{\star}$ is the crystallized mass fraction of the model.

happens at higher $T_{\mathrm{eff}}$ for larger $M_{\star}$. In the case of the sequence with $M_{\star}=1.10 M_{\odot}$, crystallization and phase separation occurs for effective temperatures characteristic of the ZZ Ceti instability strip. Figure 2 illustrates the situation for a model of this sequence at $T_{\text {eff }} \sim 11600 \mathrm{~K}$. An abrupt step is visible in the chemical profiles at the border of the rehomogeneization region, at $-\log \left(1-M_{\mathrm{r}} / M_{\star}\right) \sim 1$. This step is clearly reflected in the form of the Ledoux term $B$ as a very sharp peak, which is also visible in the shape of the Brunt-Väisälä frequency. Another notable feature in the chemical profiles is the triple interface of ${ }^{12} \mathrm{C},{ }^{16} \mathrm{O}$, and ${ }^{20} \mathrm{Ne}$, located at $1 \lesssim-\log \left(1-M_{\mathrm{r}} / M_{\star}\right) \lesssim 1.5$. This interface gives place to a bump in the Brunt-Väisälä frequency. From $-\log \left(1-M_{\mathrm{r}} / M_{\star}\right)$ approximately two to approximately five, there is a smooth transition region of ${ }^{4} \mathrm{He},{ }^{12} \mathrm{C}$, and ${ }^{16} \mathrm{O}$, which leads to a very extended and almost negligible contribution to the Ledoux term $B$ and the Brunt-Väisälä frequency. Finally, the remainder outstanding feature in the Ledoux term is the contribution of the ${ }^{4} \mathrm{He} /{ }^{1} \mathrm{H}$ chemical transition region, which is translated into the shape of $N^{2}$ as a bump at $-\log \left(1-M_{\mathrm{r}} / M_{\star}\right) \sim 6$. The magnitude of this bump increases for the more massive models. We note that the innermost peak in $B$ is due to the small step located exactly at the edge of the crystallized core. This also appears in the BruntVäisälä frequency as a small peak. In contrast with what occurs with the previously described features of $N^{2}$, the peak at the boundary of the crystallized core has no consequences for the pulsation spectrum because it is beyond the propagation zone of the $g$-modes.

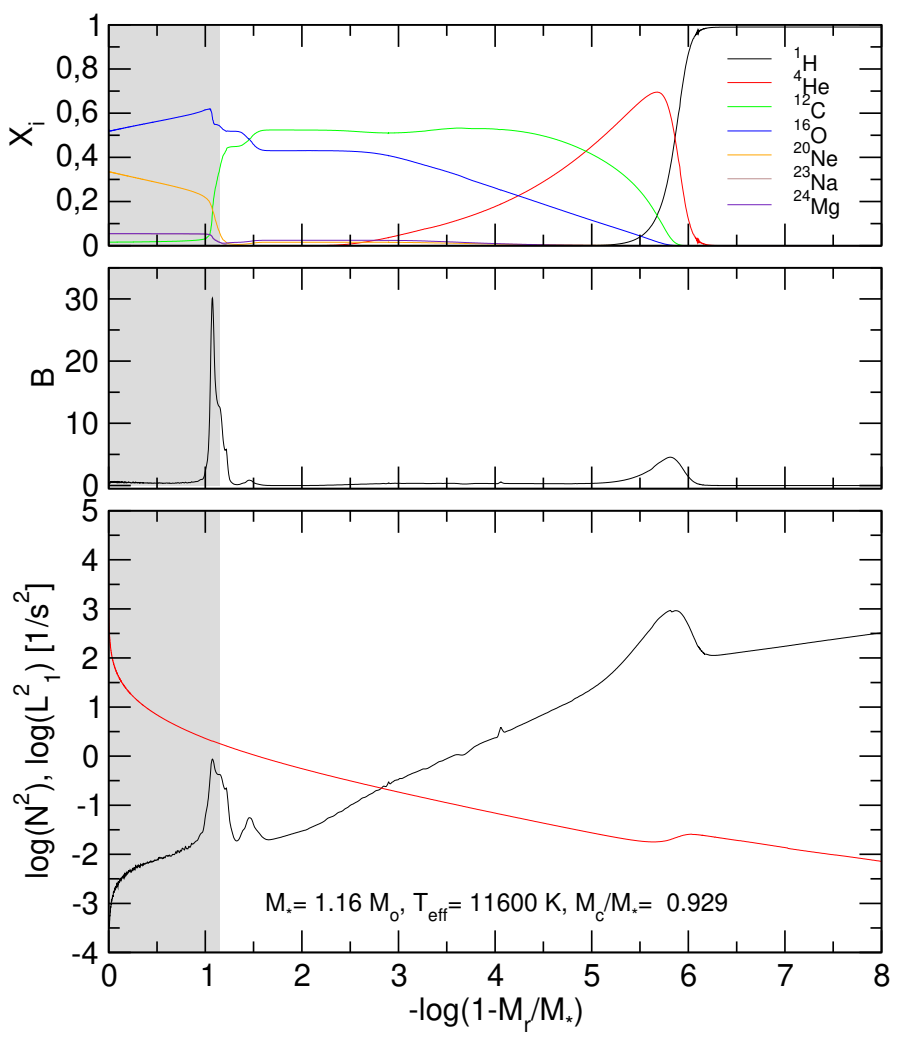

Fig. 3. Same as in Fig. 2, but for an ONe-core WD model with $M_{\star}=1.16 M_{\odot}$.

In the case of the $1.16 M_{\odot}$ model (Fig. 3), at $T_{\text {eff }} \sim 11600 \mathrm{~K}$ the chemical redistribution of ${ }^{16} \mathrm{O}$ and ${ }^{20} \mathrm{Ne}$ by phase separation has already finished, and the resulting step at the border of the rehomogeneized region $\left(-\log \left(1-M_{r} / M_{\star}\right) \sim 1\right)$ is contained in the crystallized region. Consequently, the associated bump in the Brunt-Väisälä frequency is irrelevant for the properties of $g$ modes, which do not propagate in that region. In this way, the Brunt-Väisälä frequency has only one bump which is able to inflict mode trapping effects on the modes, that is the bump at $-\log \left(1-M_{r} / M_{\star}\right) \sim 6$ due to the ${ }^{4} \mathrm{He} /{ }^{1} \mathrm{H}$ chemical interface.

The situation is similar for the models with $M_{\star}=1.22 M_{\odot}$ (Fig. 4) and $M_{\star}=1.29 M_{\odot}$ (Fig. 5). Indeed, in these cases the bump in the Brunt-Väisälä frequency due to the triple transition region of ${ }^{12} \mathrm{C},{ }^{16} \mathrm{O}$, and ${ }^{20} \mathrm{Ne}$ is excluded from the propagation cavity of $g$ modes and the period spectrum is affected only by the ${ }^{4} \mathrm{He} /{ }^{1} \mathrm{H}$ chemical transition region.

Finally, a detailed inspection of the central panels of Figs. $2-5$ in the region of the crystallized core reveals that the Ledoux term $B$ has non-zero values as a result of the spatial variation of the abundances of ${ }^{16} \mathrm{O}$ and ${ }^{20} \mathrm{Ne}$ that are due to the chemical redistribution by phase separation. This small contribution of $B$ to the Brunt-Väisälä frequency, which is more notorious for the more massive models, is not relevant for their pulsation properties because the eigenfunctions of $g$-modes are excluded from the solid region.

\subsection{Impact of crystallization on the pulsation spectrum}

In this section we analyze the pulsation properties of our set of ONe-core ultra-massive WD models. To do this, we compare the impact of crystallization by comparing the results for the cases $\mathrm{NC}, \mathrm{LH}$, and $\mathrm{LH}+\mathrm{PS}$. 

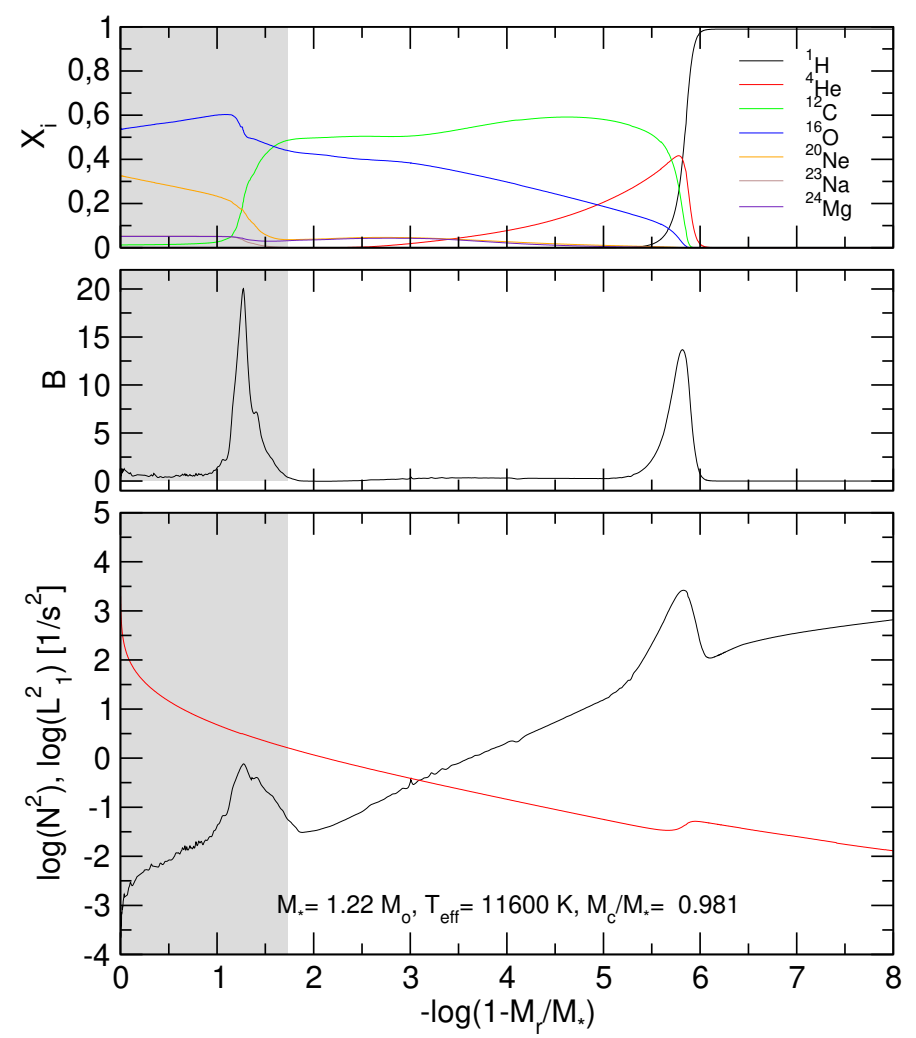

Fig. 4. Same as in Fig. 2, but for an ONe-core WD model with $M_{\star}=1.22 M_{\odot}$.

We begin by examining the asymptotic period spacing of our sequences, computed according to Eqs. (2) and (3). In Fig. 6 we depict $\Delta \Pi_{\ell}^{a}$ for $\ell=1$ modes in terms of $T_{\text {eff }}$ for the ONe-core WD evolutionary sequences with masses $1.10,1.16$, 1.22 , and $1.29 M_{\odot}$. The results correspond to the case in which crystallization was computed taking into account latent heat release and chemical rehomogenization caused by phase separation ( $\mathrm{LH}+\mathrm{PS}$ case). In the case of the 1.22 and $1.29 M_{\odot}$ sequences, the models have almost $99 \%$ of their mass crystallized throughout the $\mathrm{ZZ}$ Ceti instability strip. According to Eqs. (2) and (3), the dependence of $\Delta \Pi_{\ell}^{a}$ on the Brunt-Väisälä frequency is such that the asymptotic period spacing is larger when the mass and/or effective temperature of the model is lower. This trend is clearly visible in Fig. 6. The higher values of $\Delta \Pi_{\ell}^{a}$ for lower $M_{\star}$ comes from the dependence $N \propto g$ (Eq. (4)), where $g$ is the local gravity $\left(g \propto M_{\star} / R_{\star}^{2}\right)$. On the other hand, the higher values of $\Delta \Pi_{\ell}^{a}$ for lower $T_{\text {eff }}$ result from the dependence $N \propto \sqrt{\chi}_{T}$ (Eq. (4)), with $\chi_{T} \rightarrow 0$ for increasing electronic degeneracy $(T \rightarrow 0)$.

A remarkable feature of $\Delta \Pi_{\ell}^{a}$ is the abrupt change in the slope of the curves at certain effective temperatures. In the case of the $1.10 M_{\odot}$ sequence this happens at $T_{\text {eff }} \sim 10000 \mathrm{~K}$, for the sequence with $1.16 M_{\odot}$ this occurs at $T_{\text {eff }} \sim 12000 \mathrm{~K}$, for $1.22 M_{\odot}$ at $T_{\text {eff }} \sim 14000 \mathrm{~K}$, and for $1.29 M_{\odot}$ at $T_{\text {eff }} \sim 17000 \mathrm{~K}$ (not visible in Fig. 6). The change in the slope is due to the advance of the crystallization front. At a given point of the evolution, this front surpasses the position of the triple interface of ${ }^{12} \mathrm{C},{ }^{16} \mathrm{O}$, and ${ }^{20} \mathrm{Ne}$. This implies that the bump in the Brunt-Väisälä frequency associated to this interface ends up being inside the crystallized region and therefore excluded from the resonant cavity. According to Eq. (3), the integral decreases abruptly when this happens, so that the asymptotic period spacing experiences a sudden growth (Eq. (2)).
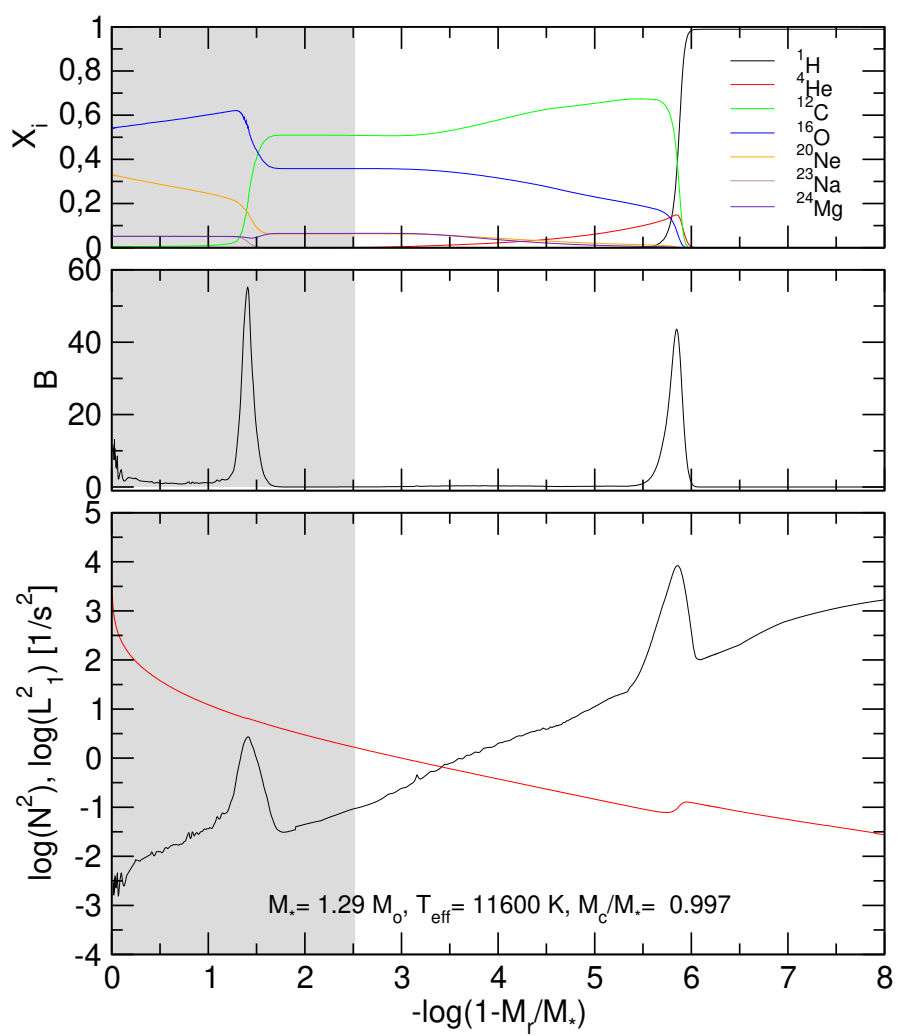

Fig. 5. Same as in Fig. 2, but for an ONe-core WD model with $M_{\star}=1.29 M_{\odot}$.

In order to show the different expected behavior of the asymptotic period spacing when we consider the $\mathrm{NC}, \mathrm{LH}$, and $\mathrm{LH}+\mathrm{PS}$ treatments, we display in Fig. $7 \Delta \Pi_{\ell=1}^{a}$ in terms of the effective temperature for the ONe-core WD evolutionary sequence with mass $M_{\star}=1.29 M_{\odot}$. For this sequence, the onset of crystallization occurs at $T_{\text {eff }} \sim 37500 \mathrm{~K}$. For higher effective temperatures, the three curves coincide. When the model cools below this effective temperature, the curves of the asymptotic period spacing corresponding to the $\mathrm{LH}$ and $\mathrm{LH}+\mathrm{PS}$ cases begin to separate from the curve associated with the NC case. The reason is, as described before, that when crystallization begins, the lower limit of the integral in Eq. (3) coincides with the radius of the crystallization boundary, which continuously moves outward as the star cools down. Thus, the fraction of crystallized mass increases and the integral in Eq. (3) decreases, leading to an increase in $\Delta \Pi_{\rho}^{a}$ (Eq. (2)). The asymptotic period spacing for the LH and LH+PS cases is always longer than for the $\mathrm{NC}$ case, also at the stages along the ZZ Ceti instability strip, where $\Delta \Pi_{\ell}^{a}$ is $\sim 4$ s shorter when crystallization is not considered. On the other hand, the value of $\Delta \Pi_{\ell}^{a}$ for the LH case is slightly higher than for the LH+PS case, the larger difference (that amounts to $\sim 1.5 \mathrm{~s}$ ) being at the ZZ Ceti instability strip. The case of the sequence with mass $M_{\star}=1.29 M_{\odot}$ is representative of the results for the sequences with masses $1.10,1.16$, and $1.22 M_{\odot}$, which are not shown for brevity. Note the abrupt increase of $\Delta \Pi_{\ell}^{a}$ at effective temperatures around $\sim 17000 \mathrm{~K}$ for both the LH and LH+PS cases, due to the reasons explained in detail in the previous paragraph.

An important diagnostic tool for studying the mode-trapping properties in pulsating WDs is the $\Delta \Pi-\Pi$ diagram, in which the separation of periods with consecutive radial order $k$ is plotted in terms of the pulsation periods. A notable consequence of modetrapping phenomena is that if we consider a fixed $T_{\text {eff }}$ value, the separation between consecutive periods has departures from the 


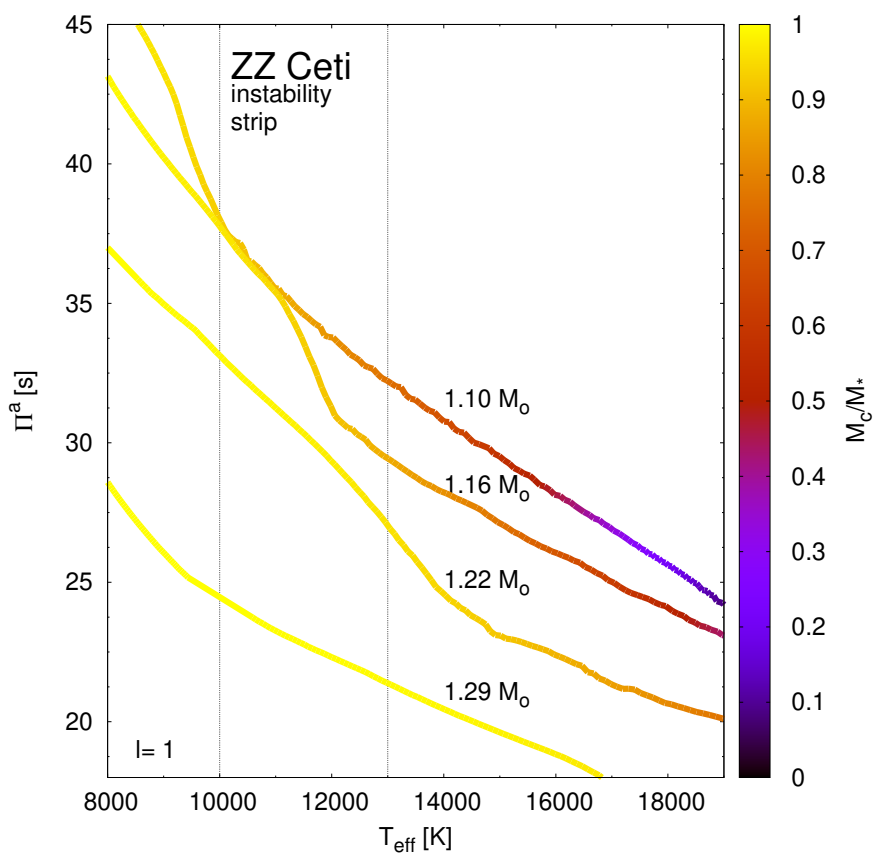

Fig. 6. Asymptotic period spacing, $\Delta \Pi^{a}$, for dipole modes $(\ell=1)$ as a function of the effective temperature for the ONe evolutionary cooling sequences with masses $1.10,1.16,1.22$, and $1.29 M_{\odot}$. Crystallization was computed taking into account latent heat release and chemical rehomogenization caused by phase separation. The palette of colors corresponds to the fraction of crystallized mass $\left(M_{\mathcal{c}} / M_{\star}\right)$. Vertical dotted lines show the $T_{\text {eff }}$ interval of the ZZ Ceti instability strip.

mean period spacing. This is clearly emphasized in the $\Delta \Pi-\Pi$ diagram shown in the right panels of Fig. 8, which show the forward period spacing $\left(\Delta \Pi_{k} \equiv \Pi_{k+1}-\Pi_{k}\right.$. $)$ in terms of the periods of $\ell=1$ pulsation modes for a $1.29 M_{\odot}$ ONe-core WD model at $T_{\text {eff }} \sim 11600 \mathrm{~K}$ for the $\mathrm{LH}+\mathrm{PC}$ case (right), the $\mathrm{LH}$ case (middle panel) and the NC case (left panel). In all the cases, $\Delta \Pi_{k}$ exhibits maxima and minima typical of WD models harboring one or more chemical transition regions. The relationship between the values of the asymptotic periods spacing for the three cases follows the trend seen in Fig. 6. In particular, for the NC case, $\Delta \Pi_{\ell}^{a}$ is markedly smaller than for the LH and $\mathrm{LH}+\mathrm{PS}$ cases because the propagation cavity where the integral of the Eq. (3) is computed is quite greater when crystallization is neglected and $g$ modes can propagate to regions closer to the center of the star.

Regarding the mode-trapping patterns, the LH and LH+PS cases (central and right panels of Fig. 8) look very similar each other. They consist of alternating maxima and minima $\Delta \Pi_{k}$, typical of WD models harboring a unique chemical interface. This is expected, given that for a model at $T_{\text {eff }} \sim 11600 \mathrm{~K}$, the core chemical interfaces are inside the crystallized region, and the only interface remaining at the propagation cavity is that of ${ }^{4} \mathrm{He} /{ }^{1} \mathrm{H}$. The mode-trapping pattern associated to the NC case (left panel of Fig. 8), on the other hand, is a little more complex and this is due to the fact that there is more than one chemical interface playing a role in the mode-trapping properties of the model.

In the lower panels of Fig. 8 we plot the logarithm of the pulsation kinetic energy in terms of periods for $1.29 M_{\odot}$. We employed the usual normalization condition, that is, that the value of the radial Eigenfunction $\left(y_{1}=\xi_{r} / r\right)$ is set to 1 at the surface of the model. The kinetic energy values plotted against the periods exhibit virtually the same behavior in the three cases considered.

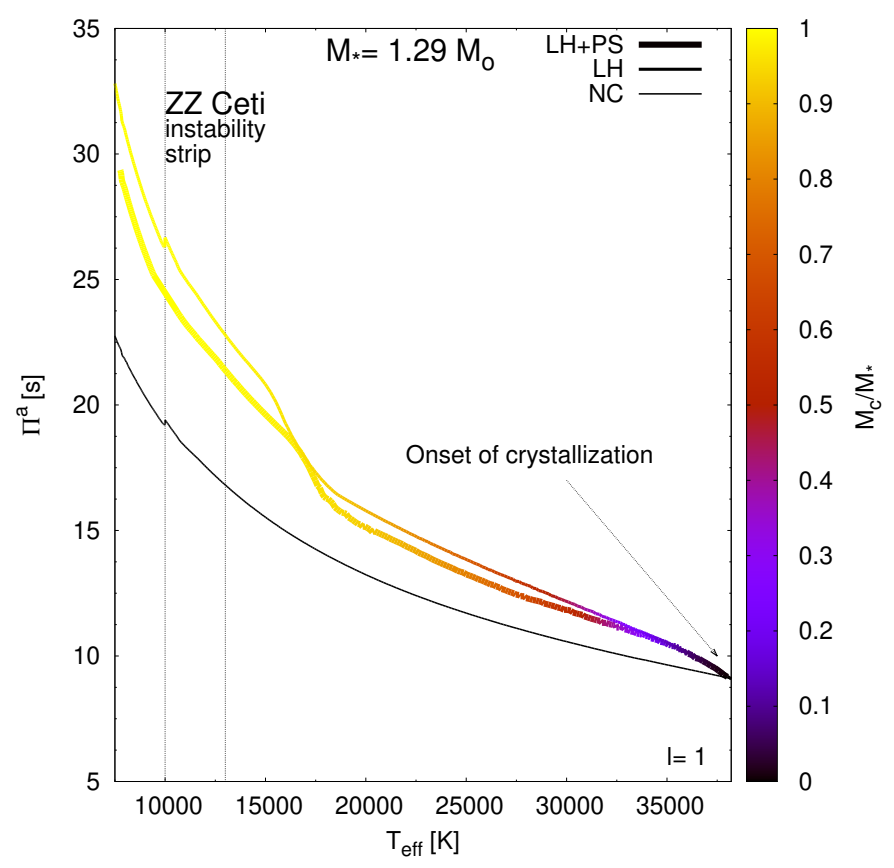

Fig. 7. Dipole $(\ell=1)$ asymptotic period spacing as a function of the effective temperature for the ONe evolutionary cooling sequence with mass $M_{\star}=1.29 M_{\odot}$. The thick curve corresponds to the case in which latent heat and chemical redistribution caused by phase separation have been taken into account during crystallization (LH+PS case), the intermediate-thickness curve displays the case in which chemical redistribution caused by phase separation has been neglected (LH case), and the thin curve is associated with the case in which crystallization has been neglected (NC case). The palette of colors corresponds to the fraction of crystallized mass $\left(M_{\mathrm{c}} / M_{\star}\right)$. Vertical dotted lines show the $T_{\text {eff }}$ interval of the ZZ Ceti instability strip.

Figure 9 depicts the same as Fig. 8, but for WD models with $T_{\text {eff }} \sim 11600 \mathrm{~K}$ and $M_{\star}=1.10 M_{\odot}$. The mode-trapping behaviour associated to these models is quite similar to that described for the $M_{\star}=1.29 M_{\odot}$ models. In particular, the period-spacing patterns for $\mathrm{LH}$ and $\mathrm{LH}+\mathrm{PS}$ cases are virtually indistinguishable, whether we look at the $\Delta \Pi_{k}$ values or the $\log \left(E_{\text {kin }}\right)$ values. On the other hand, the amplitudes of mode trapping are larger for the $\mathrm{NC}$ case.

From the results shown in Figs. 8 and 9, we conclude that it would not be feasible to distinguish the cases considered, that is $\mathrm{NC}$, LH, or LH+PS from the pulsation patterns of real ZZ Ceti stars, in particular from the period spacing values.

\subsection{Comparison with CO-core WD models}

The mass limit that separates the WDs harboring CO cores from those with ONe cores is a matter of debate. It is therefore interesting to compare the pulsation properties of WDs with CO cores and ONe cores with the same stellar mass. The aim of this analysis is to explore the possibility of using WD asteroseismology to distinguish between the two types of objects. This has been precisely the goal of the study in Córsico et al. (2004), who compared the pulsation properties of a $1.06 M_{\odot}$ WD model with $\mathrm{CO}$ core and a model with the same mass, but a core made of $\mathrm{O}$ and $\mathrm{Ne}$. Here, we re-examine the topic by taking into account the chemical rehomogenization caused by phase separation. This piece of physics was ignored in Córsico et al. (2004) for the ONe-core WD model employed in that work. 

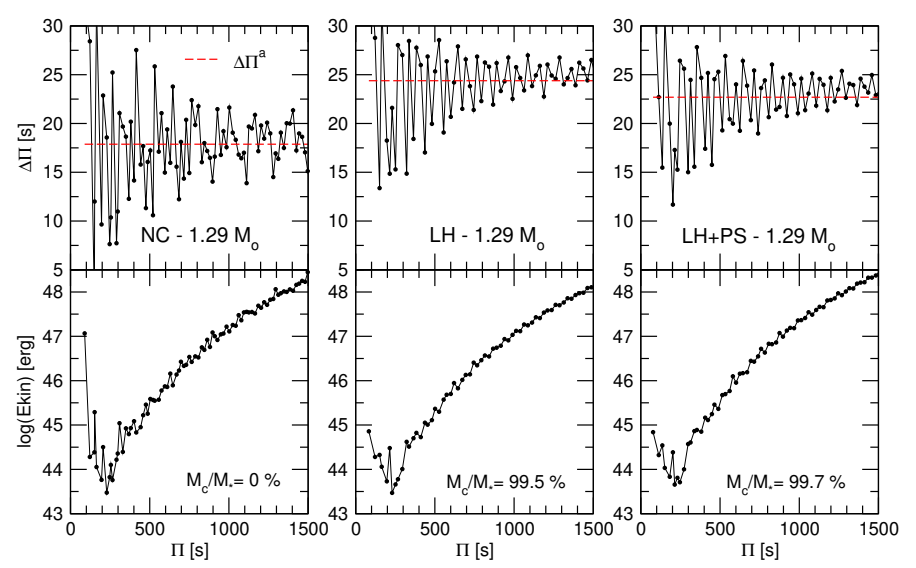

Fig. 8. Forward period spacing $(\Delta \Pi$, upper panels $)$ and logarithm of the kinetic energy ( $E_{\mathrm{kin}}$, lower panels) in terms of the periods of $\ell=1$ pulsation modes for a $1.29 M_{\odot}$ ONe-core WD model at $T_{\text {eff }} \sim 11600 \mathrm{~K}$. Left panels: case in which crystallization was not taken into account (NC case). Central panels: same quantities for the situation in which crystallization was considered but phase separation was not (LH case). Right panels: case in which both crystallization and phase separation were taken into account (LH+PS case). In the three cases, the percentage of the crystallized mass is indicated. In the upper panels, horizontal red dashed lines correspond to the asymptotic period spacing.
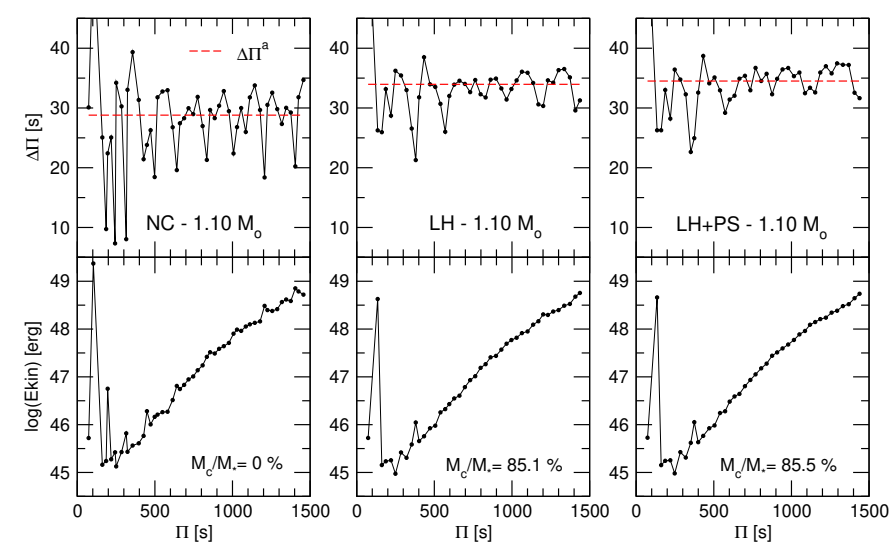

Fig. 9. Same as Fig. 8, but for an ONe-core WD model with $M_{\star}=$ $1.10 M_{\odot}$.

We computed an additional evolutionary sequence of WD models with $M_{\star}=1.10 M_{\odot}$ and a core made of ${ }^{12} \mathrm{C}$ and ${ }^{16} \mathrm{O}$ for our analysis here, taking into account latent heat release and chemical rehomogenization caused by phase separation during crystallization (LH+PS treatment). We employed the azeotropic phase diagram for a mixture of $\mathrm{C}$ and $\mathrm{O}$ reported by Horowitz et al. (2010). For this sequence, we computed the adiabatic pulsation periods of $g$-modes in the range of periods observed in ZZ Ceti stars, as well as the asymptotic period spacing. In Fig. 10 we show the dipole $(\ell=1)$ asymptotic period spacing as a function of $T_{\text {eff }}$ for the ONe evolutionary cooling sequence and the $\mathrm{CO}$ evolutionary cooling sequence with stellar mass $M_{\star}=1.10 M_{\odot}$. Both sequences were computed taking into account latent heat release and chemical redistribution due to phase separation during crystallization ( $\mathrm{LH}+\mathrm{PS}$ case). The figure shows that the asymptotic period spacing for both sequences is very similar, showing a modest difference of $\sim 2 \mathrm{~s}$ at the beginning of the ZZ Ceti instability strip (blue edge), and becoming virtually the same towards the red edge. This is a somewhat expected result given that the asymptotic period spacing of $g$-modes in ZZ Ceti stars basically

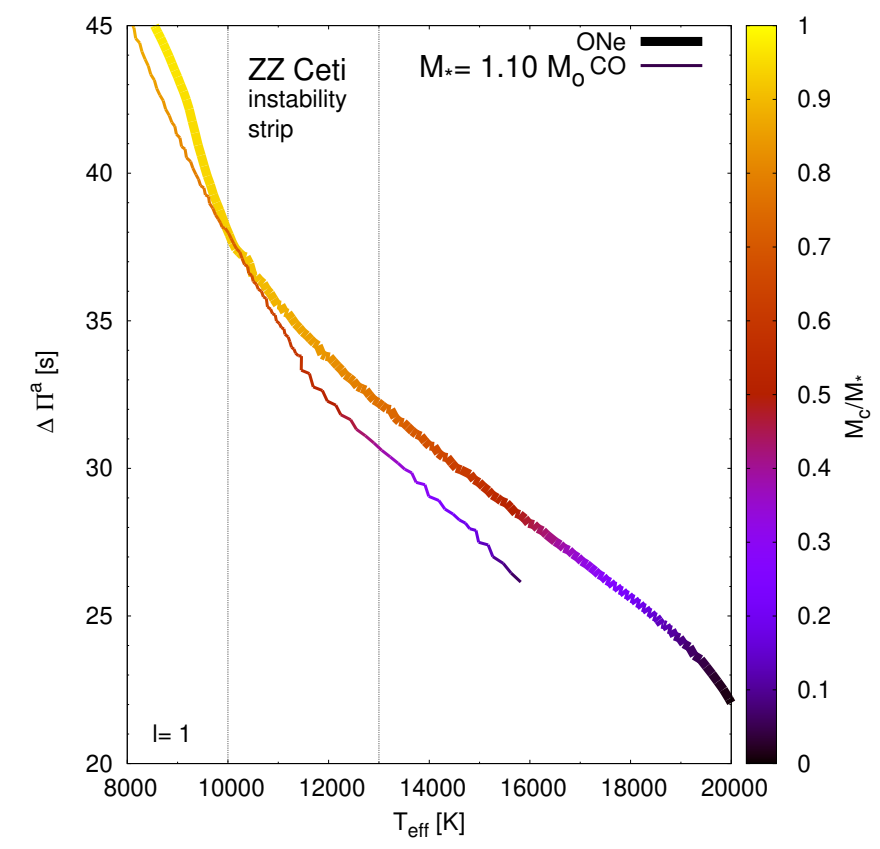

Fig. 10. Dipole $(\ell=1)$ asymptotic period spacing as a function of the effective temperature for the ONe evolutionary cooling sequence (thick curve) and the $\mathrm{CO}$ evolutionary cooling sequence with mass $M_{\star}=1.10 M_{\odot}$. Both sequences were computed taking into account latent heat release and chemical redistribution caused by phase separation during crystallization ( $\mathrm{LH}+\mathrm{PS}$ case). Vertical dotted lines show the $T_{\text {eff }}$ interval of the ZZ Ceti instability strip.

depends on the stellar mass, the thickness of the $\mathrm{H}$ envelope, and the effective temperature, and these three parameters are the same for both sequences. We note, however, that by virtue of the different core chemical compositions, the degree of crystallization for a given $T_{\text {eff }}$ is different for both types of WD models. We conclude that the asymptotic period spacing would not be a useful quantity for distinguishing ZZ Cetis harboring $\mathrm{CO}$ cores from those with cores made of ONe.

An alternative way to distinguish WDs with CO cores from those with ONe cores for a fixed stellar mass is to examine the period-spacing values in terms of the periods. This has been the approach employed by Córsico et al. (2004). To this aim, we selected a $1.10 M_{\odot}$ CO-core template WD model at an effective temperature at the middle of the ZZ Ceti instability strip $\left(T_{\text {eff }} \sim 11600 \mathrm{~K}\right)$. In Fig. 11 we show the abundances by mass of ${ }^{1} \mathrm{H},{ }^{4} \mathrm{He},{ }^{12} \mathrm{C}$, and ${ }^{16} \mathrm{O}$ as a function of the fractional mass, the Ledoux term $B$, and the logarithm of the squared BruntVäisälä and Lamb frequencies of this template CO-core WD model, with a crystallized mass of $\sim 56 \%$. In Fig. 12 we plot the period spacing and the kinetic oscillation energy in terms of the periods for the CO-core template model and its counterpart ONe-core WD model (whose properties are shown in Fig. 2). In the central panels we depict the situation for $T_{\text {eff }} \sim 11600 \mathrm{~K}$. Notably, $\Delta \Pi^{a}$ is almost the same for both models, in agreement with the discussion in the previous paragraph. However, the period-spacing distribution is qualitatively different the two sets of models. Indeed, the pattern exhibited by the CO-core WD models is characterized by larger minima in $\Delta \Pi_{k}$ values for periods longer than $\sim 500 \mathrm{~s}$, as compared with the pattern shown by the ONe-core WD models. Interestingly enough, this result is the opposed to that of Córsico et al. (2004), who found that their ONe-core WD model showed stronger mode-trapping features than their CO-core WD model. This discrepancy in the results 

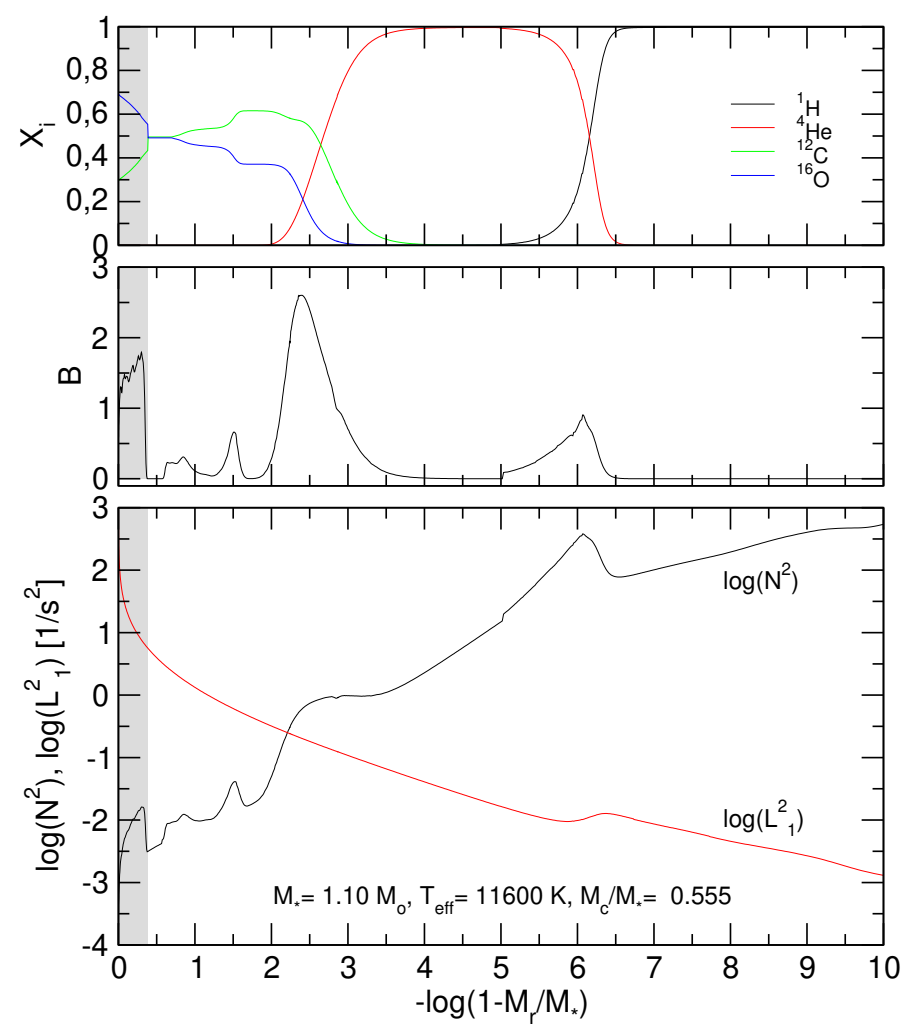

Fig. 11. Abundances by mass of ${ }^{1} \mathrm{H},{ }^{4} \mathrm{He},{ }^{12} \mathrm{C}$, and ${ }^{16} \mathrm{O}$ as a function of the fractional mass (upper panel), the Ledoux term $B$ (middle panel), and the logarithm of the squared Brunt-Väisälä and Lamb frequencies (lower panel), corresponding to a CO-core WD model with $M_{\star}=1.10 M_{\odot}, \log \left(M_{\mathrm{H}} / M_{\star}\right)=-6$, and $T_{\text {eff }} \sim 11600 \mathrm{~K}$. Latent heat release and chemical redistribution caused by phase separation have been taken into account during crystallization ( $\mathrm{LH}+\mathrm{PS}$ case). The gray area marks the domain of crystallization. $M_{\mathrm{c}} / M_{\star}$ is the fraction of the crystallized mass of the model.

arises because Córsico et al. (2004) considered chemical rehomogenization caused by phase separation in the case of the WD model with CO core, but not in the case of the WD model with ONe core, whereas we here take this effect into account for both types of chemical compositions. In addition, the chemical structure of our CO-core WD models largely differ from that of the models adopted by Córsico et al. (2004).

We conclude that the forward period spacing could be employed to differenciate cores made of ${ }^{12} \mathrm{C}$ and ${ }^{16} \mathrm{O}$, or ${ }^{16} \mathrm{O}$ and ${ }^{20} \mathrm{Ne}$, provided that a sufficient number of $g$ modes with consecutive radial order are detected. Another plausible way to determine whether an ultra-massive ZZ Ceti star has a CO core or a ONe core is to carry out detailed asteroseismological studies involving period-to-period comparisons of real stars employing two sets of ultra-massive WD models, one of them characterized by CO-core WDs and the other one with ONe-core WDs. This analysis is beyond the scope of this study and will be the focus of a future work.

\section{Summary and conclusions}

We have assessed the adiabatic pulsation properties of ultramassive H-rich WDs with ONe cores on the basis of full evolutionary models that incorporate the most recent physical inputs governing the progenitor and the WD evolution. This investigation constitutes a substantial improvement over results reported by Córsico et al. (2004) in several aspects. On one hand, the
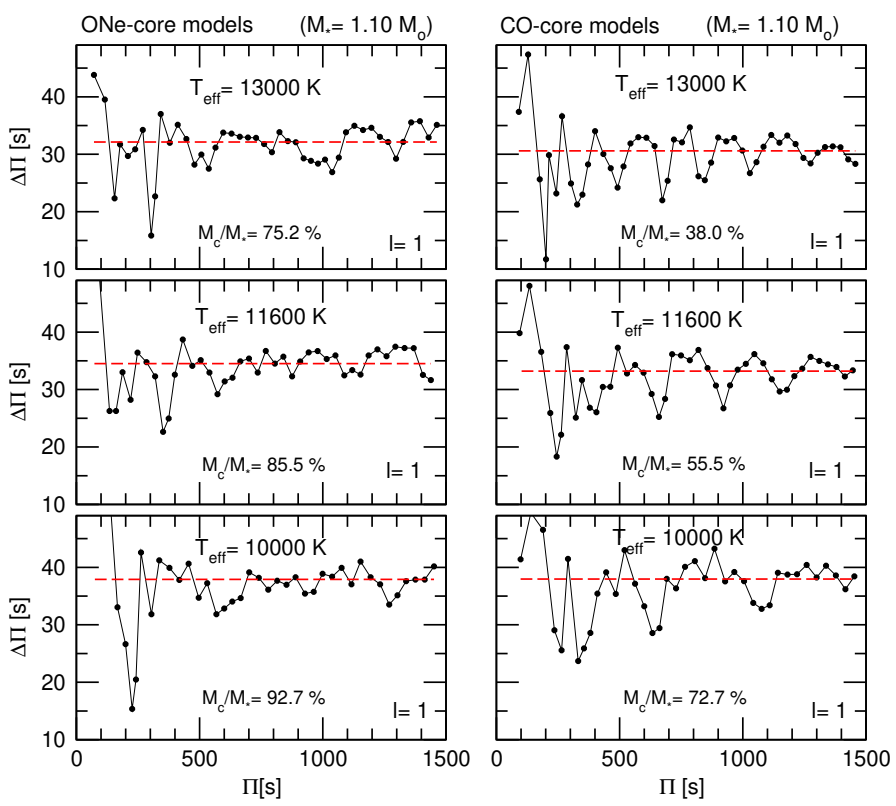

Fig. 12. Forward period spacing $(\Delta \Pi)$ in terms of the periods of $\ell=1$ pulsation modes for a $1.10 M_{\odot} \mathrm{WD}$ model at $T_{\text {eff }} \sim 13000 \mathrm{~K}$ (upper panels), $11600 \mathrm{~K}$ (middle panels), and $\sim 10000 \mathrm{~K}$ (lower panels) with an ONe core (left panels) and a CO core (right panels). In both set of models, latent-heat release and chemical redistribution caused by phase separation have been taken into account during crystallization (LH+PS case). The percentage of the crystallized mass are indicated. The horizontal dashed red line is the asymptotic period spacing.

chemical profiles of our WD models are consistent with the predictions of the progenitor evolution through the S-AGB phase for all the WD sequences with different stellar masses considered. On the other hand, during WD evolution, we have taken into account for the first time the changes in the core chemical composition resulting from phase separation upon crystallization using phase diagrams suitable for ${ }^{16} \mathrm{O}$ and ${ }^{20} \mathrm{Ne}$ plasmas. Finally, element diffusion was included for all model sequences, from the beginning of the WD cooling track. Element diffusion smoothes the inner chemical profiles, which strongly affects the run of the Brunt-Väisälä frequency, and thus the period spectrum and mode trapping properties.

We assessed the pulsational properties of our models by computing their dipole $(\ell=1)$ and quadrupole $(\ell=2) g$-mode period spectra for a wide range of effective temperatures, and in particular, for the $T_{\text {eff }}$ interval defining the ZZ Ceti instability strip. Because our models are very massive $\left(1.10 \leq M_{\star} /\right.$ $\left.M_{\odot} \leq 1.29\right)$, when they reach the ZZ Ceti instability strip, a very large portion of their mass is in a crystalline phase. In order to explore the impact of crystallization on their pulsation properties in detail, we considered three cases: (i) we neglected crystallization in the equilibrium models and also in computing the pulsation modes, (ii) we considered crystallization with the ensuing release of latent heat, and computed the pulsation modes adopting the hard-sphere boundary conditions, and (iii) we considered crystallization with latent heat release and also took into account phase separation that induces a chemical rehomogeneization in the liquid part surrounding the crystallized core and the eigenmodes are computed using the hard-sphere boundary conditions. We do not find appreciable differences in the pulsation spectra of our WD models for the cases we analyzed. In particular, we do find a very similar distribution of period spacings in the case in which the changes of the core chemical profiles that are due to phase separation are taken into account in comparison with the 
case in which it is ignored. In the case in which crystallization is ignored, the mode-trapping features in the periods-pacing diagram are slightly more pronnounced than in the cases in which crystallization is considered.

We also revisited the possibility of using asteroseismology to distinguish ultra-massive DA WDs harboring ONe cores from those having $\mathrm{CO}$ cores. In particular, we compared the pulsation properties (specifically, periods and period spacings) of WD models characterized by the same stellar mass $\left(1.10 M_{\odot}\right)$, but in one case having a core made of ${ }^{16} \mathrm{O}$ and ${ }^{20} \mathrm{Ne}$ and in another case a core composed by ${ }^{12} \mathrm{C}$ and ${ }^{16} \mathrm{O}$. We did not find sizeable differences in the mean period spacing at the ZZ Ceti stage. However, we find appreciable differences in the period-spacing distribution, in agreement with Córsico et al. (2004). In line with the claim of Córsico et al. (2004), we therefore conclude that periodspacing diagrams would allow to distinguishing the chemical composition of the cores of ultra-massive ZZ Ceti stars, provided that a sufficient number of $g$-mode periods with consecutive radial order are detected as to allow the construction of such period-spacing diagrams. Additionally, in order to infer the core composition of ultra-massive DA WDs, it will be profitable to carry out a detailed asteroseismic analysis using the individual periods observed in ultra-massive ZZ Ceti stars such as BPM 37093 (Kanaan et al. 1992, 2005), GD 518 (Hermes et al. 2013), and SDSS J0840+5222 (Curd et al. 2017). This will be the focus of a future work.

Acknowledgements. We wish to acknowledge the suggestions and comments of an anonymous referee that strongly improved the original version of this work. We gratefully acknowledge A. Cumming from providing us with the phase diagram and L. Siess for the chemical profiles of his models. Part of this work was supported by AGENCIA through the Programa de Modernización Tecnológica BID 1728/OC-AR, and by the PIP 112-200801-00940 grant from CONICET. This research has made use of NASA's Astrophysics Data System.

\section{References}

Abrikosov, A. A. 1961, Sov. Phys. JETP, 12, 1254

Althaus, L. G., Serenelli, A. M., Panei, J. A., et al. 2005, A\&A, 435, 631

Althaus, L. G., Córsico, A. H., Isern, J., \& García-Berro, E. 2010a, A\&ARv, 18 , 471

Althaus, L. G., Córsico, A. H., Bischoff-Kim, A., et al. 2010b, ApJ, 717, 897

Althaus, L. G., García-Berro, E., Renedo, I., et al. 2010c, ApJ, 719, 612

Althaus, L. G., Miller Bertolami, M. M., \& Córsico, A. H. 2013, A\&A, 557, A19

Althaus, L. G., Camisassa, M. E., Miller Bertolami, M. M., Córsico, A. H., \& García-Berro, E. 2015, A\&A, 576, A9

Brassard, P., \& Fontaine, G. 2005, ApJ, 622, 572

Brassard, P., Fontaine, G., Wesemael, F., Kawaler, S. D., \& Tassoul, M. 1991, ApJ, 367, 601

Camisassa, M. E., Althaus, L. G., Córsico, A. H., et al. 2018, A\&A, submitted

Cassisi, S., Potekhin, A. Y., Pietrinferni, A., Catelan, M., \& Salaris, M. 2007, ApJ, 661, 1094
Castanheira, B. G., Kepler, S. O., Kleinman, S. J., Nitta, A., \& Fraga, L. 2010, MNRAS, 405, 2561

Castanheira, B. G., Kepler, S. O., Kleinman, S. J., Nitta, A., \& Fraga, L. 2013, MNRAS, 430, 50

Córsico, A. H., \& Althaus, L. G. 2006, A\&A, 454, 863

Córsico, A. H., García-Berro, E., Althaus, L. G., \& Isern, J. 2004, A\&A, 427, 923

Córsico, A. H., Althaus, L. G., Montgomery, M. H., García-Berro, E., \& Isern, J. 2005, A\&A, 429, 277

Curd, B., Gianninas, A., Bell, K. J., et al. 2017, MNRAS, 468, 239

De Gerónimo, F. C., Althaus, L. G., Córsico, A. H., Romero, A. D., \& Kepler, S. O. 2017, A\&A, 599, A21

De Gerónimo, F. C., Althaus, L. G., Córsico, A. H., Romero, A. D., \& Kepler, S O. 2018, A\&A, 613, A46

Dziembowski, W. A. 1971, Acta Astron., 21, 289

Ferguson, J. W., Alexander, D. R., Allard, F., et al. 2005, ApJ, 623, 585

Fontaine, G., \& Brassard, P. 2008, PASP, 120, 1043

Garcia-Berro, E., \& Iben, I. 1994, ApJ, 434, 306

Garcia-Berro, E., Hernanz, M., Isern, J., \& Mochkovitch, R. 1988a, Nature, 333, 642

Garcia-Berro, E., Hernanz, M., Mochkovitch, R., \& Isern, J. 1988b, A\&A, 193, 141

García-Berro, E., Ritossa, C., \& Iben, Jr., I. 1997, ApJ, 485, 765

García-Berro, E., Torres, S., Althaus, L. G., et al. 2010, Nature, 465, 194

Hermes, J. J., Kepler, S. O., Castanheira, B. G., et al. 2013, ApJ, 771, L2

Horowitz, C. J., Schneider, A. S., \& Berry, D. K. 2010, Phys. Rev. Lett., 104, 231101

Iglesias, C. A., \& Rogers, F. J. 1996, ApJ, 464, 943

Isern, J., Mochkovitch, R., García-Berro, E., \& Hernanz, M. 1997, ApJ, 485, 308

Kanaan, A., Kepler, S. O., Giovannini, O., \& Diaz, M. 1992, ApJ, 390, L89

Kanaan, A., Nitta, A., Winget, D. E., et al. 2005, A\&A, 432, 219

Kirzhnits, D. A. 1960, Sov. Phys. JETP, 11, 365

Kleinman, S. J., Kepler, S. O., Koester, D., et al. 2013, ApJS, 204, 5

Magni, G., \& Mazzitelli, I. 1979, A\&A, 72, 134

Medin, Z., \& Cumming, A. 2010, Phys. Rev. E, 81, 036107

Metcalfe, T. S., Montgomery, M. H., \& Kanaan, A. 2004, ApJ, 605, L133

Miller Bertolami, M. M. 2016, A\&A, 588, A25

Miller Bertolami, M. M., Althaus, L. G., Unglaub, K., \& Weiss, A. 2008, A\&A, 491, 253

Montgomery, M. H., \& Winget, D. E. 1999, ApJ, 526, 976

Montgomery, M. H., Klumpe, E. W., Winget, D. E., \& Wood, M. A. 1999, ApJ, 525,482

Renedo, I., Althaus, L. G., Miller Bertolami, M. M., et al. 2010, ApJ, 717, 183

Romero, A. D., Córsico, A. H., Althaus, L. G., et al. 2012, MNRAS, 420, 1462

Romero, A. D., Kepler, S. O., Córsico, A. H., Althaus, L. G., \& Fraga, L. 2013, ApJ, 779, 58

Salaris, M., Domínguez, I., García-Berro, E., et al. 1997, ApJ, 486, 413

Salpeter, E. E. 1961, ApJ, 134, 669

Segretain, L., Chabrier, G., Hernanz, M., et al. 1994, ApJ, 434, 641

Siess, L. 2006, A\&A, 448, 717

Siess, L. 2007, A\&A, 476, 893

Siess, L. 2010, A\&A, 512, A10

Tassoul, M., Fontaine, G., \& Winget, D. E. 1990, ApJS, 72, 335

van Horn, H. M. 1968, ApJ, 151, 227

Wachlin, F. C., Miller Bertolami, M. M., \& Althaus, L. G. 2011, A\&A, 533, A139

Winget, D. E., \& Kepler, S. O. 2008, ARA\&A, 46, 157

Winget, D. E., Kepler, S. O., Campos, F., et al. 2009, ApJ, 693, L6 